Research Paper

\title{
Long Intergenic Noncoding RNA 00261 Acts as a Tumor Suppressor in Non-Small Cell Lung Cancer via Regulating miR-105/FHL1 Axis
}

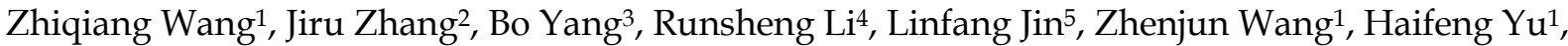 \\ Chuanxin $\mathrm{Liu}^{1}$, Yong Mao ${ }^{\circledR}$, Qingjun You ${ }^{\circledR}$ \\ 1. Department of Thoracic and Cardiovascular Surgery, Affiliated Hospital of Jiangnan University, Wuxi, Jiangsu, 214062, China. \\ 2. Department of Anesthesiology, Affiliated Hospital of Jiangnan University, Wuxi, Jiangsu, 214062, China. \\ 3. Department of Radiotherapy, Affiliated Hospital of Jiangnan University, Wuxi, Jiangsu, 214062, China. \\ 4. Department of Respiratory Medicine, Affiliated Hospital of Jiangnan University, Wuxi, Jiangsu, 214062, China. \\ 5. Department of Pathology, Affiliated Hospital of Jiangnan University, Wuxi, Jiangsu, 214062, China. \\ 6. Department of Oncology, Affiliated Hospital of Jiangnan University, Wuxi, Jiangsu, 214062, China.
}

$\triangle$ Corresponding author: Qingjun You and Yong Mao; Qingjun You, Tel.: +86051088682123; Fax: +86051085808820; Email: wxsytst@126.com. Yong Mao, Tel.: +86051088682123; Fax: +86051085808820; Email: mydoctorwx@aliyun.com.

(c) The author(s). This is an open access article distributed under the terms of the Creative Commons Attribution License (https://creativecommons.org/licenses/by/4.0/). See http://ivyspring.com/terms for full terms and conditions.

Received: 2019.06.09; Accepted: 2019.09.26; Published: 2019.10.19

\begin{abstract}
Purpose: Long noncoding RNAs (IncRNAs) have recently received more attention for their roles in tumor progression. LINC00261 was studied in this research to identify how it affects the progression of non-small cell lung cancer (NSCLC).

Methods: Firstly, the expression of LINC00261 in NSCLC cells and paired samples of NSCLC tissue was detected by RT-qPCR. Then, the associations between LINC00261 expression level and clinicopathological characteristics were evaluated. Furthermore, functional assays of cell proliferation, colony formation and transwell, as well as western blot assay, luciferase assay and RNA immunoprecipitation (RIP) assay were conducted. Afterwards, the effects of LINC00261 expression on NSCLC formation and growing were confirmed by in vivo models.

Results: As results, expression of LINC00261 was significantly down-regulated in tumor samples than that in normal samples, which was correlated with the lymphatic metastasis, tumor size, tumor stage as well as patient survival time. Knockdown of LINC00261 inhibited tumor growth and invasion ability in vitro. In addition, miR-105 was identified as a direct target of LINC00261 via mechanism experiments and its expression in tumor tissues negatively correlated to LINC00261 expression. Further experiments found that Four and expression of Half LIM domains 1 (FHLI) was negatively correlated with miR-105 but positively with LINC00261. Moreover, in vivo assays verified the overexpression of LINC00261 could suppress formation of NSCLC and regulate the expression of miR-105/FHL1 axis.

Conclusions: These results indicate that LINC00261 could suppress metastasis and proliferation of NSCLC via suppressing miR-105/FHL1 axis, which may offer a new vision for interpreting the mechanism of NSCLC development.
\end{abstract}

Key words: Non-small cell lung cancer, LINC00261, miR-105, FHL1

\section{Introduction}

As the third general cancer among human malignant tumors in 2015 [1], the incidence rate of NSCLC remains high both in male and in female worldwide. In the last decades, although molecular targeted therapy is available for NSCLC patients, only a small part of patients' benefits from those 
discovered driver mutations [2]. Therefore, further exploring the mechanisms of these genomic changes in NSCLC is urgently required.

LINC00261 has been shown to exert as a molecular biomarker in several cancers. For example, LINC00261 may be a useful biomarker of the prognosis for HCC patients, based on bioinformatics analysis [3]. Down regulation of LINC00261 may contribute to the carcinogenesis and development of laryngeal carcinoma [4]. However, it remains unknown the roles of LINC00261 in NSCLC.

Recently, plentiful studies have revealed the interaction between lncRNAs and miRNAs in several kinds of tumors which probably was associated with many oncogenes that had been known. LncRNA NEAT1 enhances the progression of colorectal cancer via competitively binding miR-34a with SIRT1 [5]. SP1-induced IncRNA-ZFAS1 is implicated in the progression of colorectal cancer via miR-150/VEGFA [6]. LncRNA DGCR5 inhibits hepatocellular carcinoma development via regulating the miR-346/KLF14 axis [7]. Shen et al. revealed that LncRNA MEG3 acts as a competing endogenous RNA to modulate HOXA11 via sponging miR-181a in myeloma [8]. LINC00888 enhances tumorigenicity of melanoma through miR-126/CRK [9]. However, the effect of this interaction on NSCLC progression is still unclear.

In this study, LINC00261 was found decreased in NSCLC samples. Besides, it inhibited invasion and proliferation in vitro. What's more, we further discovered the interaction between LINC00261 and miR-105 as well as the possible mechanism and confirmed the inhibition effect of LINC00261 on tumorgenesis of NSCLC in vivo.

\section{Materials and Methods}

\section{Clinical samples and cell lines}

60 NSCLC patients were enrolled for human tissues who received surgery at Affiliated Hospital of Jiangnan University. Before operation, written informed consent was achieved. The exclusion criteria were prior chemotherapy or radiotherapy. Tumor tissues and adjacent tissues were collected during the surgery and then immediately stored at $-80^{\circ} \mathrm{C}$. All tissues were analyzed by an experienced pathologist and the clinical data of those patients were recorded and analyzed (as shown in Table 1). The overall survival was calculated from the day of primary surgery to death or last follow-up. This study conforms to requirements of the Ethics Committee of Affiliated Hospital of Jiangnan University.

A549, SPCA1, H1975, H1299 and PC-9, and 16HBE (human normal bronchial epithelial cell) and
293T embryonic kidney cell line (American Type Culture Collection) were used in this study. Culture medium was consisted of penicillin, RPMI-1640 medium (Thermo Fisher Scientific, USA) and 10\% fetal bovine serum (FBS; Invitrogen Life Technologies). Besides, cells were cultured at $37^{\circ} \mathrm{C}$ in a humidified incubator containing $5 \% \mathrm{CO}_{2}$.

Table 1. Correlation between LINC00261 expression and clinicopathological characteristics in NSCLC patients

\begin{tabular}{|c|c|c|c|c|}
\hline \multirow[t]{2}{*}{ Characteristics } & \multirow[t]{2}{*}{ Patients } & \multicolumn{2}{|c|}{ Expression of LINC00261 } & \multirow[t]{2}{*}{$P$-value } \\
\hline & & $\begin{array}{l}\text { High- } \\
\text { LINC00261 }\end{array}$ & $\begin{array}{l}\text { Low- } \\
\text { LINC00261 }\end{array}$ & \\
\hline Total & 60 & 27 & 33 & \\
\hline Age (years) & & & & 0.693 \\
\hline$\leq 50$ & 25 & 12 & 13 & \\
\hline$>50$ & 35 & 15 & 20 & \\
\hline Gender & & & & 0.100 \\
\hline Male & 27 & 9 & 18 & \\
\hline Female & 33 & 18 & 15 & \\
\hline TNM stage & & & & $0.017^{*}$ \\
\hline I-II & 32 & 19 & 13 & \\
\hline III-IV & 28 & 8 & 20 & \\
\hline Tumor size & & & & $0.020^{*}$ \\
\hline$<3 \mathrm{~cm}$ & 30 & 18 & 12 & \\
\hline$>3 \mathrm{~cm}$ & 30 & 9 & 21 & \\
\hline Lymphatic metastasis & & & & $0.024^{*}$ \\
\hline No & 26 & 16 & 10 & \\
\hline Yes & 34 & 11 & 23 & \\
\hline
\end{tabular}

\section{Cell transfection}

After synthesized, lentiviral small hairpin RNA (shRNA) targeting LINC00261 was cloned into the pLenti-EF1a-EGFP-F2A-Puro vector (BiosettiaInc. San Diego, CA, USA). 293T cells were adopted for packaging LINC00261 lentivirus (LINC00261), the viruses and the empty vector (control). A549 cells were transfected by miRNA mimics and inhibitor provided by Genepharma Co., Ltd. (Shanghai, China). siRNA Universal Negative Control (Sigma-Aldrich) was used as controls.

\section{RNA extraction and qRT-PCR}

Total RNA was extracted from macrodissected tissue samples or cells by using TRIzol reagent (Invitrogen, USA), and then was reverse-transcribed to cDNAs via reverse Transcription Kit (Takara Biotechnology Co., Ltd., Dalian, China). The $\beta$-actin and U6 served as the internal controls for LINC00261 and mi-RNA. Available primers used for SYBR Green real-time PCR were as follows: LINC00261, forwards 5'- ACATTTGGTAGCCCGTGGAG -3' and reverse 5'TCTTCCCCGGAGAACTAGCA-3'; miR-105, forward 5'- GCCCTCG AGATACCATATCTATCCCCTTTT TCA-3', and reverse 5'- GCCGAATTC CAACC ATGAAGATACGAATTGATG-3'; $\beta$-actin, forward 5'-TTGTTACAGGAAGTCCCTTGCC-3' and reverse 5'-ATGCTATCACCTCCCCTGTGTG-3'. U6, forward 
5'-CTCGCTTCGGCAGCACATATACT-3' and reverse 5'-ACGCTTCACGAATTTGCGTGTC-3'; Thermal cycle was as follows: $30 \mathrm{sec}$ at $95^{\circ} \mathrm{C}, 5 \mathrm{sec}$ at $95^{\circ} \mathrm{C}$ for 40 cycles, $35 \mathrm{sec}$ at $60^{\circ} \mathrm{C}$.

\section{Western blot analysis}

Reagent RIPA (Beyotime) was utilized to extract protein from cells. BCA protein assay kit (Takara) was chosen for quantifying protein concentrations. The target proteins were separated by SDS-PAGE. Then they were incubated with antibodies after replaced to the polyvinylidene fluoride (PVDF) membrane. Rabbit anti- $\beta$-actin and rabbit anti-FHL1, as well as goat anti-rabbit secondary antibody were purchased from Cell Signaling Technology (CST, USA). Chemiluminescent film was applied for assessment of protein expression with Image J software.

\section{Luciferase assays and RNA immunoprecipitation (RIP) assay}

In our study, pGL3 vector (Promega) was used as a backbone for the construction of of $3^{\prime}$-UTR of FHL1 or LINC00261, wild-type (WT) 3'-UTR. Quick-change site-directed mutagenesis kit (Stratagene, Cedar Creek, USA) was adopted for site-directed mutagenesis of miR-105 binding site in FHL1 or LINC00261 3'-UTR, mutant (MUT) 3'-UTR. WT-3'-UTR or MUT-3'-UTR and miR-ctrl or miR-105 mimics was used for cell transfection. $48 \mathrm{~h}$ later, the dual luciferase reporter assay system (Promega) was utilized to perform the luciferase assays. RIP assay was performed utilizing Magna RIP RNA-Binding Protein Immunoprecipitation Kit (Millipore). Co-precipitated RNAs were detected via RT-qPCR.

\section{Ethynyl deoxyuridine (Edu) Analysis}

Proliferating cells were determined by using the 5-ethynyl-2'-deoxyuridine (EdU) abeling/detection kit (Ribobio, Guangzhou, China) according to the manufacturer's protocol. After $48 \mathrm{~h}$ of cultivation, the treated cells were incubated with $50 \mu \mathrm{M}$ EdU labeling medium for $2 \mathrm{~h}$ at $37^{\circ} \mathrm{C}$ under $5 \% \mathrm{CO}_{2}$ and fixed with $4 \%$ paraformaldehyde ( $\mathrm{pH} \mathrm{7.4)}$ for $30 \mathrm{~min}$, and then treated with $0.5 \%$ Triton $\mathrm{X}-100$ for $20 \mathrm{~min}$ at room temperature. After washing with PBS, staining with anti-EdU working solution was performed at room temperature for $30 \mathrm{~min}$. Cells were incubated with 100 $\mu \mathrm{L}$ Hoechst $33342(5 \mu \mathrm{g} / \mathrm{mL})$ at room temperature for $30 \mathrm{~min}$, followed by observation under a fluorescent microscope. The percentage of EdU-positive cells was calculated from five random fields in three wells.

\section{Colony Formation assay}

After cultured with FBS for 14 days in a six-well plate, all the cells were fixed with methanol and stained with $0.1 \%$ crystal violet. Meanwhile, numbers of colonies were counted for comparison.

\section{Wound healing assay}

A549 and SPCA1 cell lines were collected for wound healing assay. Firstly, cells were transferred into 6-well plates, and then cultured in DMEM medium overnight. After scratched with a plastic tip, cells were cultured in serum-free DMEM. Wound closure was viewed at $24 \mathrm{~h}$ and $48 \mathrm{~h}$ later. Each assay was independently repeated in triplicate.

\section{Matrigel assay}

$5 \times 10^{4}$ cells in $200 \mu \mathrm{L}$ serum-free RPMI-1640 were transformed to top chamber of an insert $(8 \mu \mathrm{m}$ pore size; Millipore) which was coated with $50 \mu \mathrm{g}$ Matrigel (BD Biosciences). And the bottom chamber was added RPMI-1640 and FBS. 48h later, after wiped by cotton swab, the top surface of chambers was immersed for $10 \mathrm{~min}$ with precooling methanol. Following were stain in crystal violet for $30 \mathrm{~min}$. Three fields were used to count the data for invasion membrane.

\section{Xenograft model}

The research was approved by the Animal Ethics Committee of Shandong University Animal Center. A549 cells $\left(6 \times 10^{5} / \mathrm{mL}\right)$ transfected with LINC00261 or control were implanted into both axillae of NOD/SCID mice (4-5 weeks old) subcutaneously. Tumor diameters were detected every 5 days. Tumor volume was calculated as the formula (Volume was calculated as length $\times$ width $^{2} \times 1 / 2$ ). Mice were sacrificed and the grafts were removed after 4 weeks. Afterwards, the tumor tissues were got and prepared for Western Blot and qRT-PCR assay to detect the protein level of FHL1 and the RNA level of miR-105 in mice from different groups.

\section{Statistical analysis}

SPSS 17.0 (SPSS, Chicago, IL, USA) was utilized to statistical analyze. Results were presented as mean \pm standard error of the mean. Chi-square test, ANOVA test and Kaplan-Meier method were selected for clinical data analysis when appropriate. The data in figures were analyzed using a paired Student's $t$ test. $\mathrm{p}$ values $<0.05$ were considered significant.

\section{Results}

\section{LINC00261 level in tissues and cells of NSCLC}

Firstly, RT-qPCR was conducted for detecting LINC00261 expression in 60 patients' tumor tissues and 5 NSCLC cells. As the results, LINC00261 was significantly downregulated in tumor tissue samples compared to the adjacent tissues (Fig. 1a). Meanwhile, LINC00261 level was lower in NSCLC cells than that in normal human bronchial epithelial 
cell (Fig. 1b). Analysis of clinicopathological features in those patients demonstrated that downregulated LINC00261 obviously correlated to tumor size, lymph node metastasis and tumor stage (Table 1). Moreover, the patients were divided into two groups according to the median expression of LINC00261, and then the survival curves were estimated using the Kaplan-Meier analysis. The results showed that patients had a better disease-free survival with higher LINC00261 level (Fig. 1c).

\section{Overexpression of LINC00261 inhibited cell proliferation and invasion in vitro}

According to LINC00261 expression in cancer cells, we chose A549 and SPCA1 cancer cells for overexpression of LINC00261. The LINC00261 lentiviruses (LINC00261) and the empty vector (control) were synthetized and transduced into these two cells. Then the LINC00261 expression was determined by qRT-PCR (Fig. 2a). Furthermore, the results of Edu assay showed that cell proliferation of NSCLC cells was inhibited after LINC00261 overexpressed (Fig. 2 b, c). Meanwhile, cancer formation assay showed that numbers of colonies were less in LINC00261 group than in control group (Fig. 2d). Then, we performed wound healing assay and transwell assay, and found that overexpressed LINC00261 suppressed NSCLC cell migration and invasion (Fig. 2 e, f). a

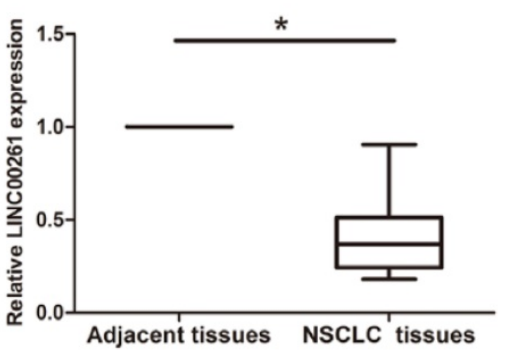

b

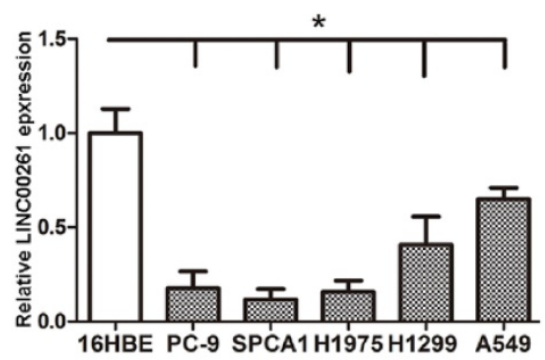

C

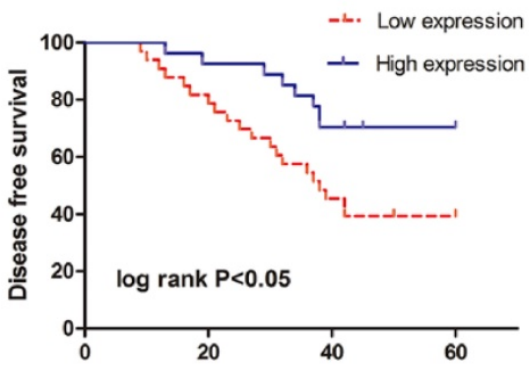

Figure 1. Expression level of LINC00261 was decreased in NSCLC tissues and cell lines, and was associated with better disease-free survival of NSCLC patients (a) LINC00261 expression was significantly decreased in the NSCLC tissues compared with adjacent tissues. (b) Expression levels of LINC00261 relative to GAPDH were determined in the human NSCLC cell lines and 16HBE (human bronchial epithelial cell) by RT-qPCR. (c) High level of LINC00261 was associated with better disease-free survival of patients with NSCLC. Data are presented as the mean \pm standard error of the mean. $* P<0.05$.

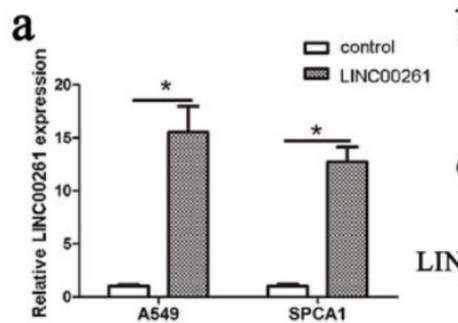

b

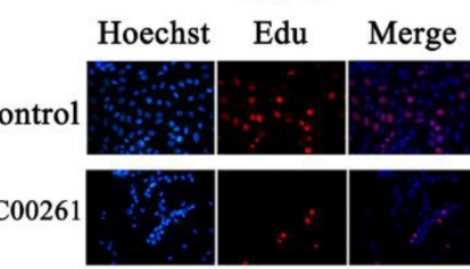

d

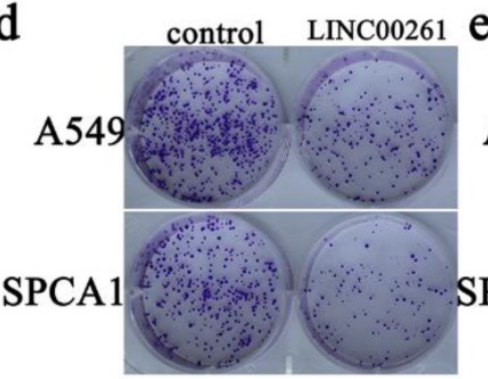

e

A549

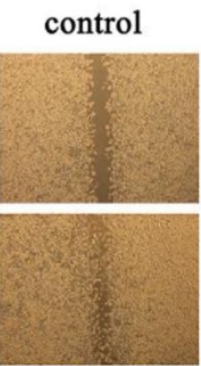

c

SPCA1

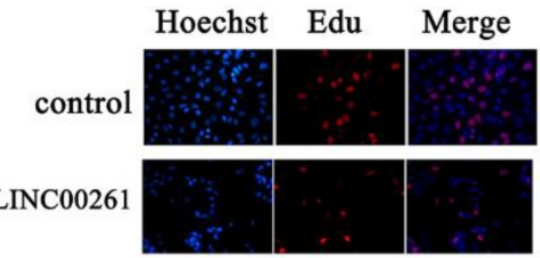

Figure 2. Overexpression of LINC00261 decreased NSCLC cell proliferation migration and invasion (a) LINC00261 expression in cancer cells transduced with empty vector (control) or LINC00261 virus (LINC00261) was detected by RT-qPCR. $\beta$-actin was used as an internal control. (b) Edu assay showed that overexpression of LINC00261 significantly decreased cell proliferation in A549 cancer cells. (c) Edu assay showed that knockdown of LINC00261 significantly decreased cell proliferation in SPCA1 cancer cells. (d) Colony formation assay demonstrated that oncogenic survival of cancer cells in LINC00261 group was significantly decreased compared with control group. (e) Wound healing assay showed that the migrated ability was obviously inhibited in LINC00261 group compared with control group. (f) Transwell assay showed that number of invaded cells in LINC00261 group was obviously reduced compared with control group. The results represent the average of three independent experiments (mean \pm standard error of the mean). $* P<0.05$, as compared with the control cells. 


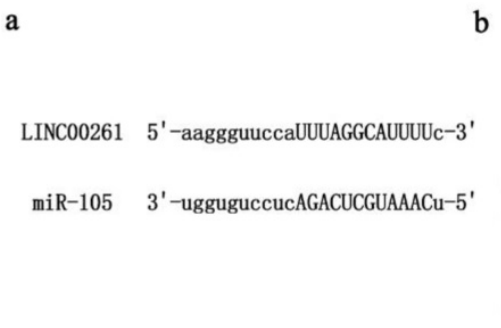

d

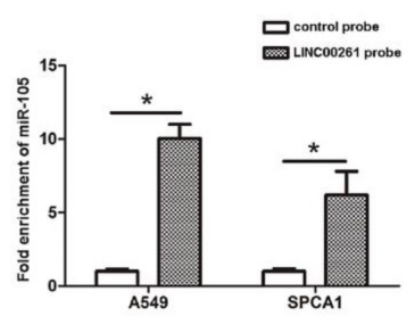

b
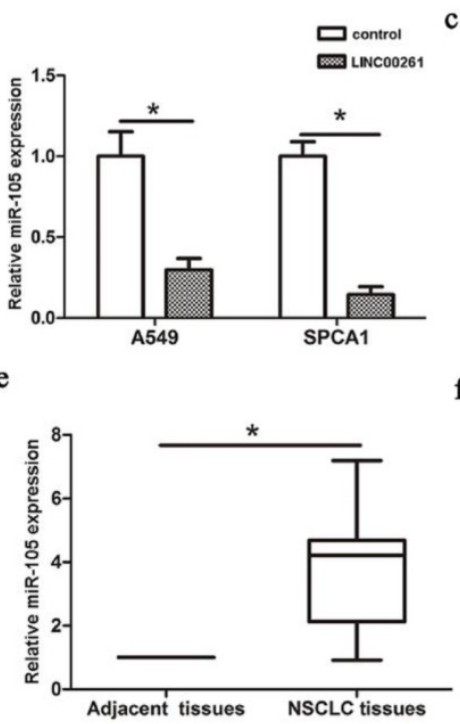

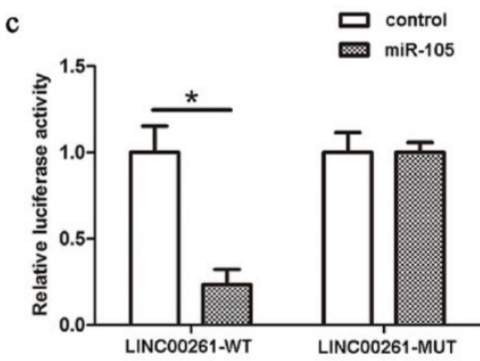

f

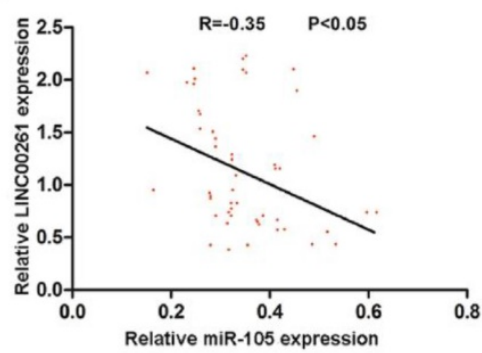

Figure 3. Interaction between LINC00261 and miR-105 (a) StarBase Predicted data was used to find the miRNAs that contained complementary base with LINC00261. (b) MiR-105 expression was decreased in LINC00261 group compared with control group. (c) Co-transfection of miR-105 and LINC00261-WT in A549 cells strongly decreased the luciferase activity, while co-transfection of miR-105 and LINC00261-MUT did not change the luciferase activity either. (d) MiR-105 was significantly enriched by RNA immunoprecipitation (RIP) assay in the LINC00261 group compared with control. (e) MiR-105 was significantly upregulated in NSCLC tissues compared with adjacent tissues. (f) The linear correlation between the expression levels of miR-105 and LINC00261 in NSCLC tissues. The results represent the average of three independent experiments Data are presented as the mean \pm standard error of the mean. $* P<0.05$.

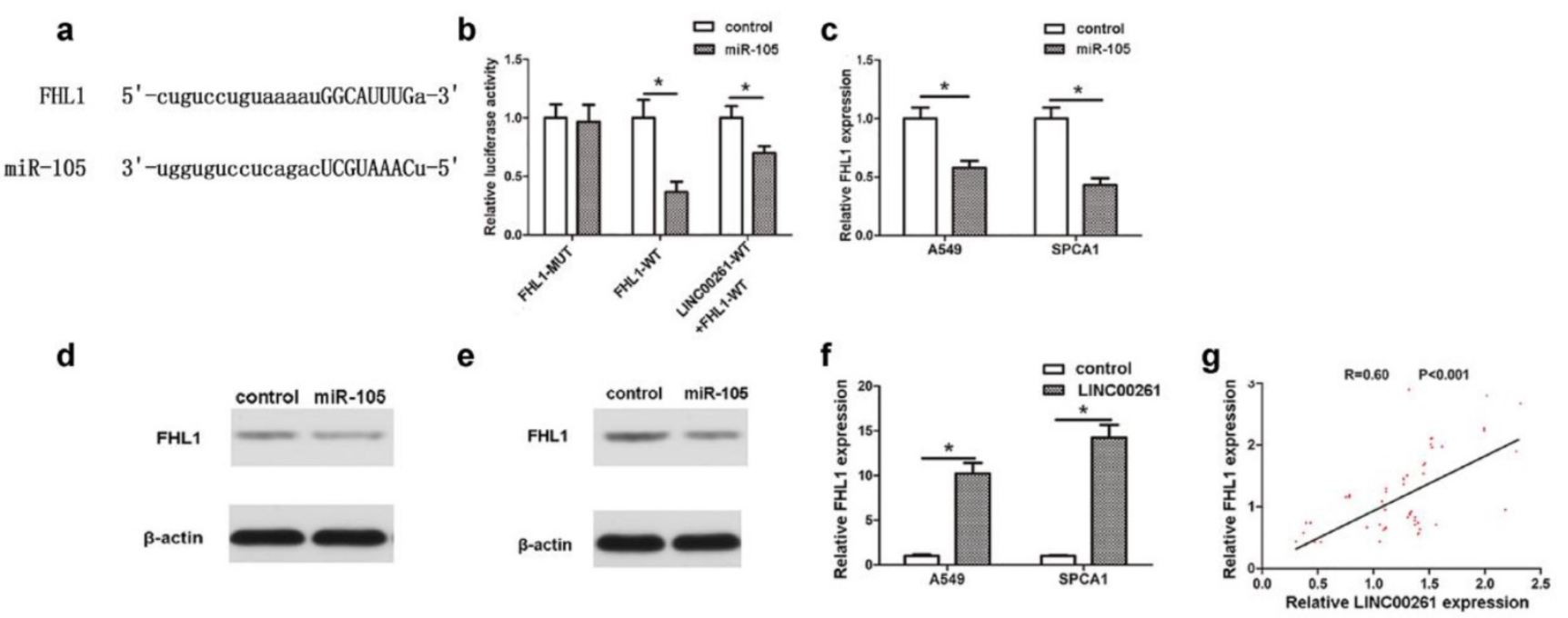

Figure 4. MiR-105/FHLI axis mediated the effect of LINC00261 on NSCLC cells (a) By using StarBase database, we predicted that FHL1 was another novel downstream target of miR-105. (b) The luciferase reporter plasmids containing the wild type 3'-UTR region or mutant 3'-UTR region of FHL1 were co-transfected into A549 cells with miR-105 or in parallel with the luciferase reporter vector LINC00261-WT. (c) FHL1 expression of cancer cells was decreased in cells transfected with miR-105 mimics compared with control cells. (d) MiR-105 mimics repressed FHL1 protein expression in A549 cells. (e) MiR-105 mimics repressed FHL1 protein expression in SPCA1 cells. (f) The expression level of FHLI in LINC00261 cells was significantly increased compared with control cells. (g) The linear correlation between the expression level of FHL1 and LINC00261 in NSCLC tissues. The results represent the average of three independent experiments. Data are presented as the mean \pm standard error of the mean. $* P<0.05$.

\section{LINC00261 inhibited NSCLC tumorigenesis via miR-105/FHLI axis}

MicroRNAs participate in biological process through their downstream targets. Jin et al. had reported that Mcl-1 was a target gene of miR-105, which contributed into NSCLC progression [11]. In this work, by using StarBase Predicted data, we predicted that FHL1 was another novel downstream target of miR-105 (Fig. 4a). The luciferase assay revealed that co-transfection of FHL1-WT and miR-105 reduced luciferase activity, which could be partly restored by LINC00261-WT (Fig. 4b). RT-PCR results further demonstrated that expression of FHL1 in NSCLC cells which transfected with miR-105 mimics was significantly downregulated (Fig. 4c). Besides, it was found that FHL1 could be inhibited at protein level by miR-105 mimics through western blot assay (Fig. $4 \mathrm{~d}$, e). To explore the interaction between LINC00261 and FHL1, FHL1 expression was detected 
in both cells and tissues. As the result, FHL1 expression of LINC00261 cells was much higher when compared to that of the control cells (Fig. 4f). The linear correlation analysis revealed that the FHL1 expression positively correlated to LINC00261 expression in NSCLC tissues (Fig. 4g).

\section{Overexpression of LINC00261 inhibited A549 cell growth in vivo}

Subcutaneous tumor mice model was established to assess cell growth. A549 cells transfected with LINC00261 or control were injected into mice subcutaneously to induce subcutaneous tumor mice model. Tumor volume was significantly smaller in LINC00261 groups compared with control groups (Fig. 5a-c). Then tumor tissues were collected to verify the expression of LINC00261 firstly. As shown in Fig. 5d, the expression of LINC00261 were overexpressed successfully in mice model of LINC00261 group. Further PCR and western blot assay showed the down-regulated of miR-105 and the higher expression of FHL1 both in RNA and protein levels in LINC00261 group (Fig. 5e-g), which revealed the effect of LINC00261 on miR-105/FHL1 axis. a

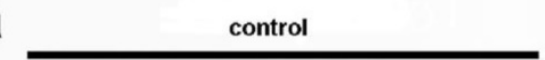

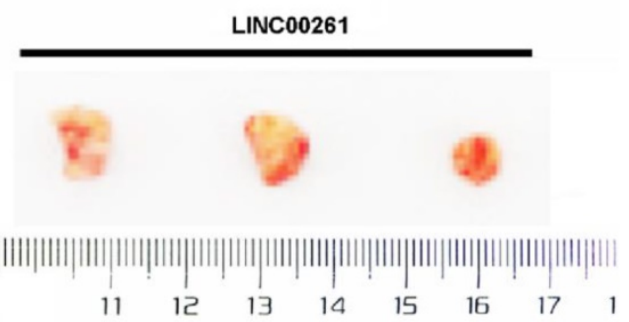

C

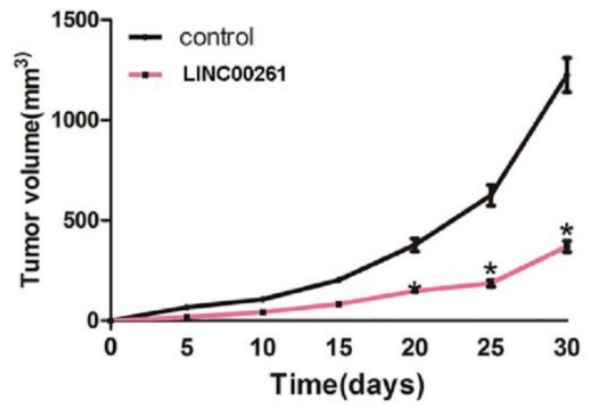

e

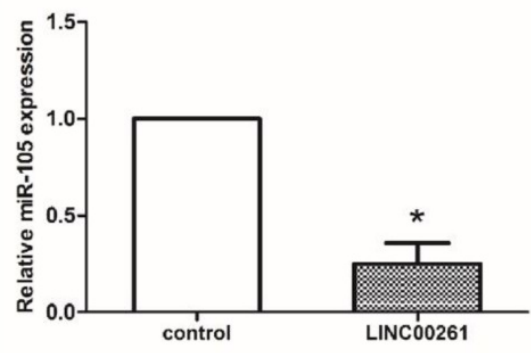

FHL1

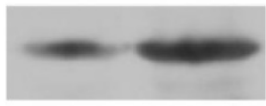

$\beta$-actin

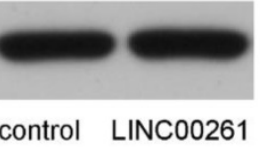

Figure 5. Overexpression of LINC00261 inhibited A549 cell growth in vivo (a) Tumor volume was determined by using calipers. (b, c) The tumor size in two groups from beginning to 1 month after model building. (d-f) The RNA expression levels of LINC00261, miR-105, as well as FHL1 were detected via PCR. ( $g$ ) Western blot assay was adopted to detect the protein levels of FHL1 in tumor tissues from two groups. The results represent the average of three independent experiments. Data are presented as the mean \pm standard error of the mean. $* P<0.05$. 


\section{Discussion}

Evidence has proved that lncRNAs participate in tumorigenesis and development. In the present study, LINC00261 was found downregulated in tissue samples and NSCLC cell lines, and the expression of LINC00261 was significant correlated with tumor size, tumor stage, lymph node metastasis and patients' prognosis. The results were consistent with previous studies, in which decreased expression of LINC00261 is a prognostic marker for NSCLC patients [12]. Zhang et al. found that LINC00261 is a novel prognostic marker for pancreatic cancer [13]. Furthermore, overexpression of LINC00261 expression could inhibit cell proliferation and invasion in NSCLC cells. Moreover, LINC00261 was also confirmed could suppress NSCLC cell growth in vivo. These data indicated that LINC00261 act as a tumor suppressor in NSCLC.

Recent studies reveal that LINC00261 participates in tumor progression in many cancers. In esophageal cancer, LINC00261 can induce chemosensitization to 5-fluorouracil via mediating methylation-dependent repression of DPYD [14]. LINC00261 controls endometrial carcinoma progression via regulating miRNA/FOXO1 [15]. Moreover, LINC00261 decreases cisplatin resistance and promotes the anti-cancer effect of cisplatin in colon cancer [16]. LINC00261 represses cell growth and migration in endometriosis [17]. Another study revealed LINC00261 inhibits cell proliferation and invasion and promotes cell apoptosis in choriocarcinoma [18]. Here, we demonstrated that LINC00261 was downregulated in NSCLC tissue samples and cells, and significantly correlated with tumor size, tumor stage, lymph node metastasis and patients' prognosis. In vitro, upregulated LINC00261 inhibited cell growth and invaded ability.

Latest studies revealed the functions of lncRNAs in NSCLC progression by binding to miRNAs. For example, LncRNA H19 acts as a ceRNA and participates in the development of NSCLC via controlling miR-107 [19]. LINC00978 enhances NSCLC cell proliferation and invasion via repressing miR-6754 [20]. It has been revealed that knockdown of lncRNA PVT1 promotes radiosensitivity in NSCLC via sponging miR-195 [21]. LINC00673 influences NSCLC proliferation, invasion and epithelial mesenchymal transition via sponging miR-150 [22]. Recent research found LINC00472 suppresses proliferation and promotes apoptosis of lung adenocarcinoma cells by modulating miR-24-3p/DEDD [23]. Our study revealed that miR-105 could directly bind to LINC00261 through a luciferase assay. And miR-105 was significantly enriched by LINC00261 RIP assay. In addition, miR-105 expression could be downregulated with overexpression of LINC00261. In NSCLC tissues, the expression of miR-105 was downregulated by LINC00261 expression. All the results above suggest that LINC00261 might inhibit tumorigenesis of NSCLC via sponging miR-105.

High miR-105 expression was associated with aggressive phenotype of colorectal cancer, and the enhanced expression of miR-105 was required for TNF- $\alpha$-induced epithelial-mesenchymal transition (EMT) [24]. Cancer-secreted miR-105 destroyed vascular endothelial barriers to promote metastasis [25]. MiR-105 promoted EMT of NSCLC via upregulating Mcl-1 [26]. However, miR-105 suppressed cell proliferation and inhibited PI3K/AKT signaling in hepatocellular carcinoma [27]. miR-105 inhibited prostate tumor growth by suppressing CDK6 levels [28]. Reduced miR-105-1 was related to poor survival of patients with NSCLC [29]. Collectively, this inconsistent finding may be due to the tumor type or contamination of normal tissue in the tumor samples or lack of samples or others. Thus, additional studies are required to clarify the role of miR-105 in NSCLC. MicroRNAs participate in biological process through their downstream targets. Jin et al. had reported that Mcl-1 was a target gene of miR-105, which contributed into NSCLC progression [11]. In this work, we identified that FHL1 was another novel downstream target of miR-105. The four and a half LIM domains (FHL) family participates in cell proliferation, apoptosis and differentiation in skeletal and cardiac muscle growth, and the member FHL1, located on chromosome Xq26 $[30,31]$. Notably, FHL1 as a tumor suppressor gene is reduced in the malignant cancers, including gastric $[32,33]$, breast, kidney and prostate cancers [34]. Niu et al. confirmed that FHL1 was downregulation and has an inhibitory role in the progression of lung cancer [35]. miRNAs can regulate biological behaviors by targeting one or more genes, which indicates the complicated processes of tumors are owing to not only one molecular but also two or more factors, even a network. Thus, further understanding the regulatory mechanism of multiple molecules in tumors progression is needed.

\section{Conclusion}

Above data identified that in NSCLC tissues, LINC00261 was remarkably downregulated and was better related to disease-free survival of NSCLC patients. Besides, LINC00261 could inhibit cell proliferation and invasion through miR-105/FHL1 axis both confirmed in vitro and in vivo. These findings suggested that LINC00261 might contribute to 
therapy for NSCLC as a candidate target.

\section{Acknowledgement}

Project supported by Natural Science Foundation of Jiangsu Province of China (Grant Number BK20161141); and the Revitalize and defend the key talent's subsidy project in science and education of department of public health of Jiangsu Province, China (Grant Number QNRC2016156).

\section{Competing Interests}

The authors have declared that no competing interest exists.

\section{References}

1. Siegel RL, Miller KD, Jemal A. Cancer statistics, 2015. CA: a cancer journal for clinicians. 2015; 65: 5-29.

2. Torre LA, Bray F, Siegel RL, Ferlay J, Lortet-Tieulent J, Jemal A. Global cancer statistics, 2012. CA: a cancer journal for clinicians. 2015; 65: 87-108.

3. Sui J, Miao Y, Han J, Nan H, Shen B, Zhang X, et al. Systematic analyses of a novel lncRNA-associated signature as the prognostic biomarker for Hepatocellular Carcinoma. Cancer medicine. 2018. [Epub ahead of print]

4. Zhang CM, Gao W, Wu YY, Zhao QL, Chen B, Liu QQ, et al. [The expression of long non-coding RNA LINC00261 in laryngeal carcinoma tissue and their clinical significance]. Lin Chung Er Bi Yan Hou Tou Jing Wai Ke Za Zhi. 2017; 31: $68-71$.

5. Luo Y, Chen JJ, Lv Q, Qin J, Huang YZ, Yu MH, et al. Long non-coding RNA NEAT1 promotes colorectal cancer progression by competitively binding miR-34a with SIRT1 and enhancing the Wnt/ beta-catenin signaling pathway. Cancer letters. 2018. 440-441:11-22

6. Chen X, Zeng $\mathrm{K}, \mathrm{Xu} \mathrm{M}, \mathrm{Hu} X$, Liu X, Xu T, et al. SP1-induced IncRNA-ZFAS1 contributes to colorectal cancer progression via the miR-150-5p/VEGFA axis. Cell death \& disease. 2018; 9: 982

7. Wang YG, Liu J, Shi M, Chen FX. LncRNA DGCR5 represses the development of hepatocellular carcinoma by targeting the miR-346/KLF14 axis. J Cell Physiol. 2018. 234(1):572-580

8. Shen X, Bai H, Zhu H, Yan Q, Yang Y, Yu W, et al. Long Non-Coding RNA MEG3 Functions as a Competing Endogenous RNA to Regulate HOXA11 Expression by Sponging miR-181a in Multiple Myeloma. Cellular physiology and biochemistry : international journal of experimental cellular physiology, biochemistry, and pharmacology. 2018; 49: 87-100.

9. Lu W, Tao X, Fan Y, Tang Y, Xu X, Fan S, et al. LINC00888 promoted tumorigenicity of melanoma via miR-126/CRK signaling axis. Onco Targets Ther. 2018; 11: 4431-42.

10. Yang JH, Li JH, Shao P, Zhou H, Chen YQ, Qu LH. starBase: a database for exploring microRNA-mRNA interaction maps from Argonaute CLIP-Seq and Degradome-Seq data. Nucleic Acids Research. 2011; 39: D202.

11. Jin $\mathrm{X}, \mathrm{Yu} \mathrm{Y}$, Zou $\mathrm{Q}$, Wang $\mathrm{M}$, Cui $\mathrm{Y}$, Xie J, et al. MicroRNA-105 promotes epithelial-mesenchymal transition of nonsmall lung cancer cells through upregulating Mcl-1. Journal of cellular biochemistry. 2018. 120(4):5880-5888

12. Liu $\mathrm{Y}, \mathrm{Xiao} \mathrm{N}, \mathrm{Xu} \mathrm{SF}$. Decreased expression of long non-coding RNA LINC00261 is a prognostic marker for patients with non-small cell lung cancer: a preliminary study. European Review for Medical \& Pharmacological Sciences. 2017; 21: 5691.

13. Zhang B, Li C, Sun Z. Long non-coding RNA LINC00346, LINC00578, LINC00673, LINC00671, LINC00261, and SNHG9 are novel prognostic markers for pancreatic cancer. American journal of translational research. 2018; 10: 2648-58.

14. Lin K, Jiang H, Zhuang SS, Qin YS, Qiu GD, She YQ, et al. Long noncoding RNA LINC00261 induces chemosensitization to 5-fluorouracil by mediating methylation-dependent repression of DPYD in human esophageal cancer. FASEB J. 2018; 33(2):1972-1988.

15. Fang Q, Sang L, Du S. Long noncoding RNA LINC00261 regulates endometrial carcinoma progression by modulating miRNA/FOXO1 expression. Cell Biochem Funct. 2018; 36: 323-30.

16. Wang ZK, Yang L, Wu LL, Mao $\mathrm{H}$, Zhou $\mathrm{YH}$, Zhang PF, et al. Long non-coding RNA LINC00261 sensitizes human colon cancer cells to cisplatin therapy. Braz J Med Biol Res. 2017; 51: e6793.

17. Sha L, Huang L, Luo $X$, Bao J, Gao L, Pan $Q$, et al. Long non-coding RNA LINC00261 inhibits cell growth and migration in endometriosis. J Obstet Gynaecol Res. 2017; 43: 1563-9.

18. Wang $Y$, Xue $K$, Guan $Y$, Jin $Y$, Liu $S$, Wang $L$, et al. Long Noncoding RNA LINC00261 Suppresses Cell Proliferation and Invasion and Promotes Cell Apoptosis in Human Choriocarcinoma. Oncology research. 2017; 25: 733-42.

19. Qian B, Wang DM, Gu XS, Zhou K, Wu J, Zhang CY, et al. LncRNA H19 serves as a ceRNA and participates in non-small cell lung cancer development by regulating microRNA-107. European review for medical and pharmacological sciences. 2018; 22: 5946-53.

20. Li X, Ren Y, Zuo T. Long noncoding RNA LINC00978 promotes cell proliferation and invasion in nonsmall cell lung cancer by inhibiting miR67545p. Molecular medicine reports. 2018. 18(5):4725-4732

21. Wu D, Li Y, Zhang H, Hu X. Knockdown of Lncrna PVT1 Enhances Radiosensitivity in Non-Small Cell Lung Cancer by Sponging Mir-195. Cellular physiology and biochemistry : international journal of experimental cellular physiology, biochemistry, and pharmacology. 2017; 42: 2453-66.

22. Lu W, Zhang $\mathrm{H}$, Niu $\mathrm{Y}, \mathrm{Wu} \mathrm{Y}$, Sun $\mathrm{W}$, Li H, et al. Long non-coding RNA linc00673 regulated non-small cell lung cancer proliferation, migration, invasion and epithelial mesenchymal transition by sponging miR-150-5p. Molecular cancer. 2017; 16: 118.

23. Su C, Shi K, Cheng X, Han Y, Li Y, Yu D, et al. Long Noncoding RNA LINC00472 Inhibits Proliferation and Promotes Apoptosis of Lung Adenocarcinoma Cells via Regulating miR-24-3p/ DEDD. Technology in cancer research \& treatment. 2018; 17: 1533033818790490.

24. Shen Z, Zhou R, Liu C, Wang Y, Zhan W, Shao Z, et al. MicroRNA-105 is involved in TNF-alpha-related tumor microenvironment enhanced colorectal cancer progression. Cell death \& disease. 2017; 8: 3213.

25. Zhou W, Fong MY, Min Y, Somlo G, Liu L, Palomares MR, et al Cancer-secreted miR-105 destroys vascular endothelial barriers to promote metastasis. Cancer cell. 2014; 25: 501-15.

26. Jin X, Yu Y, Zou Q, Wang M, Cui Y, Xie J, et al. MicroRNA-105 promotes epithelial-mesenchymal transition of nonsmall lung cancer cells through upregulating Mcl-1. Journal of cellular biochemistry. 2019; 120: 5880-8.

27. Shen G, Rong X, Zhao J, Yang X, Li H, Jiang H, et al. MicroRNA-105 suppresses cell proliferation and inhibits PI3K/AKT signaling in human hepatocellular carcinoma. Carcinogenesis. 2014; 35: 2748-55.

28. Honeywell DR, Cabrita MA, Zhao H, Dimitroulakos J, Addison CL. miR-105 inhibits prostate tumour growth by suppressing CDK6 levels. PloS one. 2013; 8: e70515.

29. Lu G, Fu D, Jia C, Chai L, Han Y, Liu J, et al. Reduced miR-105-1 levels are associated with poor survival of patients with non-small cell lung cancer. Oncology letters. 2017; 14: 7842-8.

30. Ng EK, Lee SM, Li HY, Ngai SM, Tsui SK, Waye MM, et al. Characterization of tissue-specific LIM domain protein (FHL1C) which is an alternatively spliced isoform of a human LIM-only protein (FHL1). Journal of cellular biochemistry. 2001; 82: 1-10

31. Morgan MJ, Madgwick AJ. The LIM proteins FHL1 and FHL3 are expressed differently in skeletal muscle. Biochem Biophys Res Commun. 1999; 255: 245-50.

32. Xu Y, Liu Z, Guo K. Expression of FHL1 in gastric cancer tissue and its correlation with the invasion and metastasis of gastric cancer. Mol Cell Biochem. 2012; 363: 93-9.

33. Sakashita K, Mimori K, Tanaka F, Kamohara Y, Inoue H, Sawada T, et al. Clinical significance of loss of Fhl1 expression in human gastric cancer. Ann Surg Oncol. 2008; 15: 2293-300.

34. Li X, Jia Z, Shen Y, Ichikawa H, Jarvik J, Nagele RG, et al. Coordinate suppression of Sdpr and Fhl1 expression in tumors of the breast, kidney, and prostate. Cancer science. 2008; 99: 1326-33.

35. Niu C, Liang C, Guo J, Cheng L, Zhang $\mathrm{H}$, Qin $\mathrm{X}$, et al. Downregulation and growth inhibitory role of FHL1 in lung cancer. International journal of cancer. 2012; 130: 2549-56. 